\title{
Propuesta de mejora de la satisfacción laboral en el área administrativa de una empresa importadora en Lima (2020)
}

\section{Enhancement proposal for job satisfaction within the administrative area of an import business in Lima (2020)}

Luis Alberto Chinchay-Tisoc

Universidad Norbert Wiener, Lima, Perú

lacht316@gmail.com

(iD https://orcid.org/0000-0002-6064-4890

Iris Samanta Siu Cusicanqui-Zavaleta

Universidad Norbert Wiener, Lima, Perú

iris.cusicanqui@gmail.com

(i) https://orcid.org/0000-0001-9526-7047

Roy Fernando Martínez-Quintanilla

Universidad Norbert Wiener, Lima, Perú

roy.martinez@uwiener.edu.pe

(i) https://orcid.org/0000-0002-4223-0272

Raúl Valdiviezo-López

Universidad Norbert Wiener, Lima, Perú

raul.valdiviezo@uwiener.edu.pe

(D) https://orcid.org/0000-0003-4670-3353

Recepción: 30/09/2021 | Aceptación: 15/12/2021 | Publicación: 10/01/2022

\section{Cómo citar (APA, séptima edición):}

Chinchay-Tisoc, L.A., Cusicanqui-Zavaleta, I.S.S., Martínez-Quintanilla, R. F., y ValdiviezoLópez, R. (2022). Propuesta de mejora de la satisfacción laboral en el área administrativa de una empresa importadora en Lima (2020). INNOVA Research Journal, 7(1), 77-95.

https://doi.org/10.33890/innova.v7.n1.2022.1942

\section{Resumen}

El presente estudio se planteó con la finalidad de mejorar la satisfacción laboral del área administrativa de una empresa importadora en Lima, (Perú), realizando un diagnóstico y 
explicando los factores que componen dicha satisfacción. El estudio se desarrolló bajo el enfoque mixto, considerando el sintagma holístico y los métodos inductivo y deductivo. La muestra total de la población fue de treinta (30) empleados del área administrativa, debido al número limitado de personas existentes. Las unidades informantes que accedieron a participar en la entrevista fueron tres ejecutivos de la empresa. Como instrumento se empleó el cuestionario de Sonia Palma, que consta de siete (07) subcategorías y treinta y seis (36) ítems de preguntas. Asimismo, se utilizó el diagrama de Pareto y el software IBM SPSS Statistics para procesar los datos cuantitativos, y para los datos cualitativos se empleó el software Atlas.ti; Con dicha información se procedió a realizar el diagnóstico a través de la triangulación. Finalmente, se identificaron los factores que inciden en la satisfacción laboral los cuales son la remuneración no acorde con las labores realizadas, el deterioro de las relaciones humanas y la falta de reconocimiento al mérito. Por ello, se propone mejorar la satisfacción laboral a través de la aplicación de un modelo integral que comprende entre otras herramientas, la elaboración de un informe de la escala salarial, un manual que permita identificar las habilidades de los colaboradores, un taller de relaciones humanas y un manual de reconocimiento al mérito.

Palabras claves: satisfacción laboral; motivación; relaciones humanas.

\begin{abstract}
This study has been thought to enhance the employees' job satisfaction by making a diagnosis and explaining the factors this satisfaction is composed of, and applied within the administrative department of an import company located in Lima (Peru). The research has been performed based on a mixed method, considering the holistic syntagm and the inductive and deductive methods. Only 30 employees working in the administrative department were involved in the sample due to the limited number of people there. Three executives from the company agreed to participate in the interview as reporting units. The Sonia Palma's questionnaire, which is composed of seven (7) subcategories and thirty-six questions, was used as an instrument. Likewise, the Pareto diagram and the IBM SPSS statistic software were utilized to process quantitative data, while the Atlas.ti software was used to obtained the qualitative data. Such information allowed for the diagnosis triangulation. To conclude, the insufficient remuneration for the work done, the interpersonal relationships decline and the lack of acknowledgement were identified as the factors affecting job satisfaction. Therefore, it is proposed to enhance the job satisfaction by applying a comprehensive model including, among other tools, the making of a report on the salary gap, a guide to detect the employee's skills, a workshop on interpersonal relationship and a guide for the acknowledgement of merits.
\end{abstract}

Key words: job satisfaction; motivation; human relations.

\title{
Introducción
}

En esta coyuntura actual, es innegable la preocupación que existe a nivel mundial por la satisfacción de los trabajadores, debido a que gran parte de sus empleadores se han olvidado que las organizaciones están compuestas de personas, cada una de ellas con sus propias singularidades, y por ello es muy necesario encontrar los medios que les brinden un alto grado de motivación, de ahí parte la necesidad de efectuar múltiples investigaciones científicas; por ejemplo, a nivel internacional, un estudio realizado por la Society For Human Resource Management en los 
Propuesta de mejora de la satisfacción laboral en el área administrativa de una empresa importadora en Lima (2020)

Estados Unidos sobre satisfacción laboral, determinó que el 38\% de los trabajadores de este país, se encuentran altamente satisfechos, mientras que un 51\% indicó estar satisfecho, pero en menor escala; en esta misma pesquisa se determinó la relación existente entre la satisfacción y el compromiso de los colaboradores, lo que resulta de suma importancia para el desarrollo de las organizaciones (Society For Human Resource Management, 2017, p.9). En México se realizó una investigación de metodología cualitativa, en donde los entrevistados de dos empresas de distintas ciudades, señalaron que no se les brindaba las facilidades para tener un mejor desarrollo dentro de su centro de labores, sea por negar permisos para estudiar o por no contar con oportunidades de ascenso (Sanchez y García, 2017). En otro estudio efectuado por Randstad en España, se detectó el descenso de la satisfacción laboral en un 3\%, al pasar de $74 \%$ a $71 \%$ en el primer trimestre del año 2019, de igual modo, determinaron que aquellos que gozan de educación superior tienen una mayor satisfacción que aquellos que cuentan solo con formación primaria (Randstad Workmonitor, 2019, p.1). En un estudio elaborado en Brasil, se observó que la implementación del home office ocasionó un incremento de la satisfacción laboral de hasta 85\%, asimismo, el 71\% considera que las gerencias de hoy en día requieren poner en práctica un esquema en base a resultados, y no a la asistencia presencial del colaborador; de igual manera, el trabajador se sentirá más identificado y satisfecho con la organización (Sap Consultoria, 2016, p.23).

En el Perú, luego de realizada una encuesta a un grupo de trabajadores, se observó que el $30 \%$ de encuestados está muy satisfecho con su trabajo actual, el $49 \%$ está algo satisfecho y el $21 \%$ no señala ningún grado de satisfacción; asimismo, se determinó que el $32 \%$ de profesionales encuestados indican que su empleo les permite hacer línea de carrera y desarrollarse; sin embargo, el $12 \%$ señala no tener oportunidades de progreso laboral (Lee Hecht Harrison - DBM Perú, 2018). Por otra parte, según un estudio a nivel nacional, solo un $44 \%$ de los trabajadores están satisfechos laboralmente; del mismo modo, el $74 \%$ de los encuestados si cambiarían de empleo si encontraran un trabajo que los haga sentir más felices, esto guarda concordancia con otro punto de esta encuesta, el cual es la relación entre comodidad y equilibro entre la vida y el trabajo, a lo que el $48 \%$ considera que las organizaciones no promueven estas prácticas, lo que afecta en forma directa su satisfacción (Ronald Carrer Services Group y Andina agencia peruana de noticias, 2018).

La organización materia de estudio es una empresa importadora mayorista ubicada en Lima, Perú, dedicada a la distribución y venta de accesorios de cómputo, suministros para impresoras, y tecnologías de almacenamiento. Dentro de la empresa, en el área administrativa, se identificaron tres causas que perturban la satisfacción laboral del equipo de trabajo, la primera de ellas, es que se tienen condiciones inadecuadas de trabajo, a raíz del cambio de modalidad de trabajo presencial a home office, considerando que los colaboradores han tratado de adecuar un espacio en sus domicilios, estos ambientes no cuentan con la iluminación y conexiones eléctricas necesarias, asimismo, comparten el hogar con otros integrantes de su familia que realizan otras actividades o también laboran con la modalidad de trabajo remoto, lo que aún es más incómodo; además, los colaboradores realizan tareas domésticas durante la jornada laboral. Como segunda causa se consideran los procedimientos internos deficientes debido a que no existe un control de las labores que son ejecutadas por los trabajadores, de igual modo, no hay registro de incidencias, ni metodologías de trabajo; todo ello debido a la carencia de instrumentos para la gestión de cada puesto de trabajo, así como la falta de técnicas para la mejora de procesos. Por último, la tercera causa es la sobrecarga laboral, dado que no se organizan las actividades que se deben desempeñar 
cada día, no hay una adecuada priorización de tareas, no se percibe una correcta proporción en la asignación de tareas y se ejecutan labores fuera del rango del horario laboral.

\section{Problemas}

¿Cómo se puede mejorar la satisfacción laboral dentro del área administrativa de una empresa importadora, Lima 2020?

¿Cómo es la satisfacción laboral dentro del área administrativa de una empresa importadora, Lima 2020?

¿Cuáles son los factores de mayor relevancia en la satisfacción laboral dentro del área administrativa de una empresa importadora, Lima 2020?

\section{Objetivo}

Proponer estrategias de mejora que puedan incrementar la satisfacción laboral en el área administrativa de una empresa importadora, Lima 2020.

La presente investigación se planteó con el propósito de conocer los factores que inciden en la satisfacción laboral, diagnosticar y explicar sus componentes y con dicha información plantear un plan de mejora que permita optimizar el funcionamiento, producción y rendimiento de los colaboradores del área administrativa de una empresa importadora a través del sentirse plenamente satisfechos.

\section{Marco teórico}

Para el estudio se emplearon cuatro teorías, la teoría de los dos factores de Herzberg, quien manifestaba que el nivel contrario de la satisfacción, no sería la insatisfacción, sino la carencia de satisfacción; de igual modo, el nivel contrario de la insatisfacción, no sería la satisfacción, sino la inexistencia de insatisfacción (Chiavenato, 2009); asimismo, consideraba que la satisfacción era generada por el aspecto motivacional, esto en relación a que los colaboradores sienten la necesidad de ser reconocidos; y la insatisfacción, era generada por los factores higiénicos, que están relacionados a las condiciones de trabajo, el ambiente laboral, la relación con sus compañeros, las remuneraciones, entre otros elementos externos que afectan al trabajador (Chiavenato, 2007; Rodríguez, 2019). Por ende, esta teoría permitió identificar el factor extrínseco que es el ambiente y el intrínseco que es la motivación, y de ese modo, abordar la necesidad de los trabajadores por contar con las condiciones apropiadas para cumplir con sus funciones, y comprender las razones por las que dichos factores los han afectado.

En segundo lugar, la teoría de las relaciones humanas, donde predomina el concepto de organismo social, sosteniendo que toda organización está constituida no solo de elementos de corte material, sino básicamente de personas, las cuales tienen sentido de pertenencia en varios grupos sociales y por ello, se les tiene que otorgar mucha atención (Chiavenato, 2006; Dávila 2001). Además, Elton Mayo en conjunto con otros autores pertenecientes a la escuela humanista, fueron los que fomentaron la necesidad de hacer un cambio de estructura para lograr organizaciones de éxito, de ese modo surgieron las ciencias sociales, se contempló el liderazgo como base de 
autoridad, la motivación se daba a través de compensaciones de corte social, y se reemplazaron los conceptos de fatiga física por el de cansancio psicológico, y comportamiento individual por el de desempeño de grupo (Chiavenato, 2007). Por todo ello, la teoría contribuyó en el estudio de la falta de reconocimiento, las labores rutinarias y la falta de equidad en las tareas asignadas y otros aspectos que pueden ser incluidos en esta idea de hombre social, es decir, el requisito de las personas por desear relacionarse con los demás.

En tercer lugar, la teoría de la jerarquía de necesidades de Maslow, la cual señalaba que el ser humano busca alcanzar una escala de necesidades, iniciando por las de nivel primario y conforme logre satisfacerlas, irá en búsqueda de las demás necesidades de seguridad, sociales, de estima y, por último, de autorrealización; ello puede comprenderse mejor como una pirámide, donde en la base se encuentran las necesidades fisiológicas y en la cúspide se ubica la autorrealización (Chiavenato, 2006; Maslow, 1991). Por otra parte, para la gran mayoría de personas es muy difícil alcanzar los cinco niveles planteados en la teoría, es por ello, que un número muy considerable de individuos permanecerán en los últimos niveles de esta pirámide e inclusive presentarán dificultades para obtener una satisfacción adecuada (Chiavenato, 2006). Por lo descrito, esta teoría fue muy útil para entender la problemática de los colaboradores que vienen siendo afectados por la variación de condiciones laborales como consecuencia de la pandemia, impactando en la satisfacción de todas sus necesidades.

Y, en cuarto lugar, la teoría de la discrepancia, planteada por Edwin Locke, la cual formula que las empresas y los trabajadores emplean un rango de jerarquía en sus procedimientos, lineamientos, dimensiones, valores, aspectos, forma de actuar, etc.; de igual modo, el autor, toma como base que el grado de satisfacción laboral está relacionado a la valoración total que se brinda en función a lo percibido dentro de la organización, es decir, tanto lo que el colaborador valora de su trabajo como los puntos clave que son valorados por la empresa. Por lo tanto, cuando se presenta una discrepancia sustancial entre empleado y empleador sobre lo que se le brinda una mayor valoración, se disparan los índices de insatisfacción laboral. Para contrarrestar ello, Locke propuso tres elementos que ayudan a resolver las discrepancias presentadas; el primero es la satisfacción con las dimensiones del trabajo, en donde se trata la evaluación afectiva de todas las dimensiones individuales relacionadas al trabajo; el segundo elemento, es la descripción de las dimensiones, en donde se analizan las percepciones no afectivas, es decir, aquellas que se relacionan con las especificaciones y detalles del trabajo; por último, el tercer elemento, es la relevancia de las dimensiones, como cuyo nombre lo describe, revisa la importancia que cada dimensión de trabajo tiene para el colaborador (Locke, 1976). Por lo mencionado, esta teoría permitió al estudio comprender de mejor forma la relación entre lo percibido por los trabajadores y por la empresa, entendiendo cuáles son aquellos puntos donde se discrepa y por ende que factores son los que inciden en la satisfacción laboral.

\section{Metodología}

En esta investigación se empleó el método inductivo, el cual permite una visión continua de un problema porque comprende de lo específico hacia lo común, es decir, se fundamente en examinar, dar detalle y producir conclusiones; por esta razón, facilitó el procesamiento de los datos obtenidos correspondientes al cualitativo (Del Cid, Méndez, y Sandoval, 2011; Hernández, Fernández y Baptista, 2014). Del mismo modo, se aplicó el método deductivo, el cual busca 
encontrar respuestas específicas, partiendo de las conclusiones generales, explorando afirmaciones, axiomas, entre otros que sean de una fuente conocida y tengan valor; por esta razón, este método sirvió para certificar lo planteado en este trabajo a través del estudio de los datos (Bernal, 2010; Del Cid, Méndez, y Sandoval, 2011).

El enfoque empleado es el mixto, puesto que permite considerar una asociación de procedimientos experimentales, metódicos, de conocimientos y de análisis crítico en una investigación la cual requiere analizar e integrar los datos de carácter cualitativo y cuantitativo; es por esta razón, que el uso del enfoque mixto logró que este estudio contenga un margen más amplio en sus alcances y obtener mayor claridad y comprensión en el tema a estudiar, al considerar ambos perfiles de investigación y no limitarse a un solo punto de vista. (Carhuancho, Nolazco, Sicheri, Guerrero y Casana, 2019; Hernández, Fernández y Baptista, 2014).

La investigación fue proyectiva, porque se facilita una secuencia ordenada e indaga a fondo cualquier posible escenario o materia de análisis, entiende la concepción del estudio, la elección de los instrumentos que deben utilizarse, así como las técnicas a emplear en la obtención de resultados, concluyendo con un proyecto de mejora estructurado que contenga acciones, lineamientos y planes a ejecutar; por esta razón, su aplicación en este estudio brindó las herramientas necesarias para conseguir una propuesta de solución que sea útil (Carhuancho et al., 2019). Por otra parte, el nivel de investigación empleado fue el comprensivo, el cual busca examinar el caso propuesto en relación con otras situaciones de mayor dimensión y bajo esta premisa se realiza la investigación holística; debido a ello, su uso permitió entender claramente los puntos que abarcan todo el estudio (Carhuancho et al., 2019; Hurtado, 2000). Asimismo, el sintagma considerado es el holístico, porque permitió la recopilación de toda la información que el estudio del problema requirió, facilitando el generar opciones de mejora; para ello, se necesitó la comprensión reflexiva y el análisis de las ideas presentadas, de esta manera se puede lograr la formulación de alternativas de conocimiento que resulten innovadoras (Carhuancho et al., 2019).

El diseño empleado en este estudio fue el explicativo secuencial, debido a que presenta dos etapas, en la primera se busca y examinan todos los elementos cuantitativos, y en la segunda se recolectan y analizan los datos cualitativos. Estas fases se complementan una vez los hallazgos sean utilizados para crear y sustentar el estudio llevado a cabo; por esta razón, este diseño fue de utilidad para la investigación al permitir integrar las dos corrientes de datos, y comprender así, los resultados con una mayor amplitud (Hernández, Fernández y Baptista, 2014; Carhuancho et al., 2019).

La población se entiende como un conjunto de casos que presentan características en común y está compuesta por aquellos que son una fuente informativa para realizar un análisis, estás fuentes pueden ser grupos de personas y empresas u organizaciones (Carhuancho et al., 2019; Hernández, Fernández y Baptista, 2014); por otra parte, cabe indicar que este estudio la población estuvo conformada por 30 personas del área administrativa, los cuales son ocho trabajadores del departamento de finanzas, cinco de importaciones, cinco de sistemas, cuatro de recursos humanos, cuatro de contabilidad y cuatro de créditos y cobranzas. De otra parte, la muestra se conforma por un extracto de elementos que pertenecen a un grupo poblacional, es decir, la muestra es una parte de la población, reclutada para obtener información y datos para evaluar estas variables logrando los resultados requeridos para el estudio (Hernández, Fernández y Baptista, 2014; Bernal, 2010). 
De igual manera, es preciso señalar que no hubo muestra al tratarse de un reducido número de personas. Asimismo, al muestreo se le considera como un conjunto de acciones realizadas para identificar a los miembros que serán parte de una muestra, para posteriormente definir los pasos a seguir fijando el tamaño la misma, de esta forma, se escoge el método que se empleará para tomar la decisión sobre quienes serán los escogidos (Hurtado, 2000; Tecla y Garza, 1981). Reiterar que el tamaño de la muestra fue el total de la población por tratarse de un número reducido de personas.

Las unidades informantes, son quienes aportan datos de la categoría de estudio desde un punto de vista cualitativo, por ello, la información es recopilada a través de entrevistas; por otro lado, estas unidades están conformadas por los elementos o personas con características en común y de las cuales se consigue su testimonio sobre el tema de investigación (Carhuancho et al., 2019). En este caso, las unidades informantes fueron tres personas, el jefe de sistemas, un asistente de importaciones y un asistente de créditos y cobranzas, quienes brindaron los datos requeridos por el estudio y representaron equilibradamente las distintas perspectivas de los miembros del área administrativa.

\section{Tabla 1}

Matriz de categorización problema

\begin{tabular}{lll}
\hline \multicolumn{1}{c}{ Categoría } & \multicolumn{1}{c}{ Subcategorías } & \multicolumn{1}{c}{ Indicadores } \\
\hline C1: Satisfacción laboral & Condiciones físicas y/o & 1. Ambiente físico. \\
& materiales. & 2. Recursos materiales. \\
Beneficios laborales y/o & 3. Remuneración. \\
& remunerativos. & 4. Incentivo. \\
Políticas administrativas. & 5. Jornada de trabajo. \\
& 6. Horario flexible. \\
& Relaciones sociales. & 7. Clima organizacional. \\
& 8. Cultura organizacional. \\
& Desarrollo personal. & 9. Capacitación y desarrollo. \\
& 10. Ascenso. \\
& Desempeño de tareas. & 11. Actitud. \\
& 12. Comportamiento. \\
& Relación con la autoridad. & 13. Flexibilidad. \\
& 14. Relaciones interpersonales. \\
\hline
\end{tabular}

Fuente: Elaboración propia

Tabla 2

Matriz de categorización solución

\begin{tabular}{llll}
\hline \multicolumn{1}{c}{ Categoría } & \multicolumn{1}{c}{ Problema } & \multicolumn{1}{c}{ Objetivo } & \multicolumn{1}{c}{ Solución / Entregable } \\
\hline C2: Modelo super & Problema 1. & Objetivo 1. Formar super & 1.- Informe de la escala salarial. \\
teams para mejorar & Remuneración no & equipos que contribuyan & 2.- Documento de cómo identificar \\
la satisfacción & acorde con las & a la organización. & las habilidades de los colaboradores. \\
laboral
\end{tabular}

Esta obra se comparte bajo la licencia Creative Common Atribución-No Comercial 4.0 International (CC BY-NC 4.0) 


\begin{tabular}{|c|c|c|c|}
\hline Categoría & Problema & Objetivo & Solución / Entregable \\
\hline & $\begin{array}{l}\text { labores } \\
\text { realizadas. }\end{array}$ & \multirow[t]{2}{*}{$\begin{array}{l}\text { Objetivo } 2 \text {. Fortalecer las } \\
\text { relaciones humanas. }\end{array}$} & \\
\hline & $\begin{array}{l}\text { Problema } 2 . \\
\text { Deterioro de las } \\
\text { relaciones } \\
\text { humanas. }\end{array}$ & & $\begin{array}{l}\text { 3.- Manual de relaciones humanas. } \\
\text { 4.- Aporte al reglamento de trabajo. } \\
\text { 5.- Cartilla de instrucciones de } \\
\text { talleres. } \\
\text { 6.- Encuesta de satisfacción de } \\
\text { cliente interno. }\end{array}$ \\
\hline & $\begin{array}{l}\text { Problema } 3 \text {. Falta } \\
\text { de } \\
\text { reconocimiento } \\
\text { al mérito } \\
\text { individual. }\end{array}$ & $\begin{array}{l}\text { Objetivo 3. Implementar } \\
\text { el reconocimiento al } \\
\text { mérito individual. }\end{array}$ & $\begin{array}{l}\text { 7.- Modelo de aplicación de salario } \\
\text { emocional. } \\
\text { 8.- Encuesta de satisfacción laboral. } \\
\text { 9.- Manual de reconocimiento al } \\
\text { mérito. }\end{array}$ \\
\hline
\end{tabular}

Fuente: Elaboración propia

\section{Resultados y Discusión}

\section{Figura 1}

Diagrama de Pareto para el análisis de los datos cuantitativos

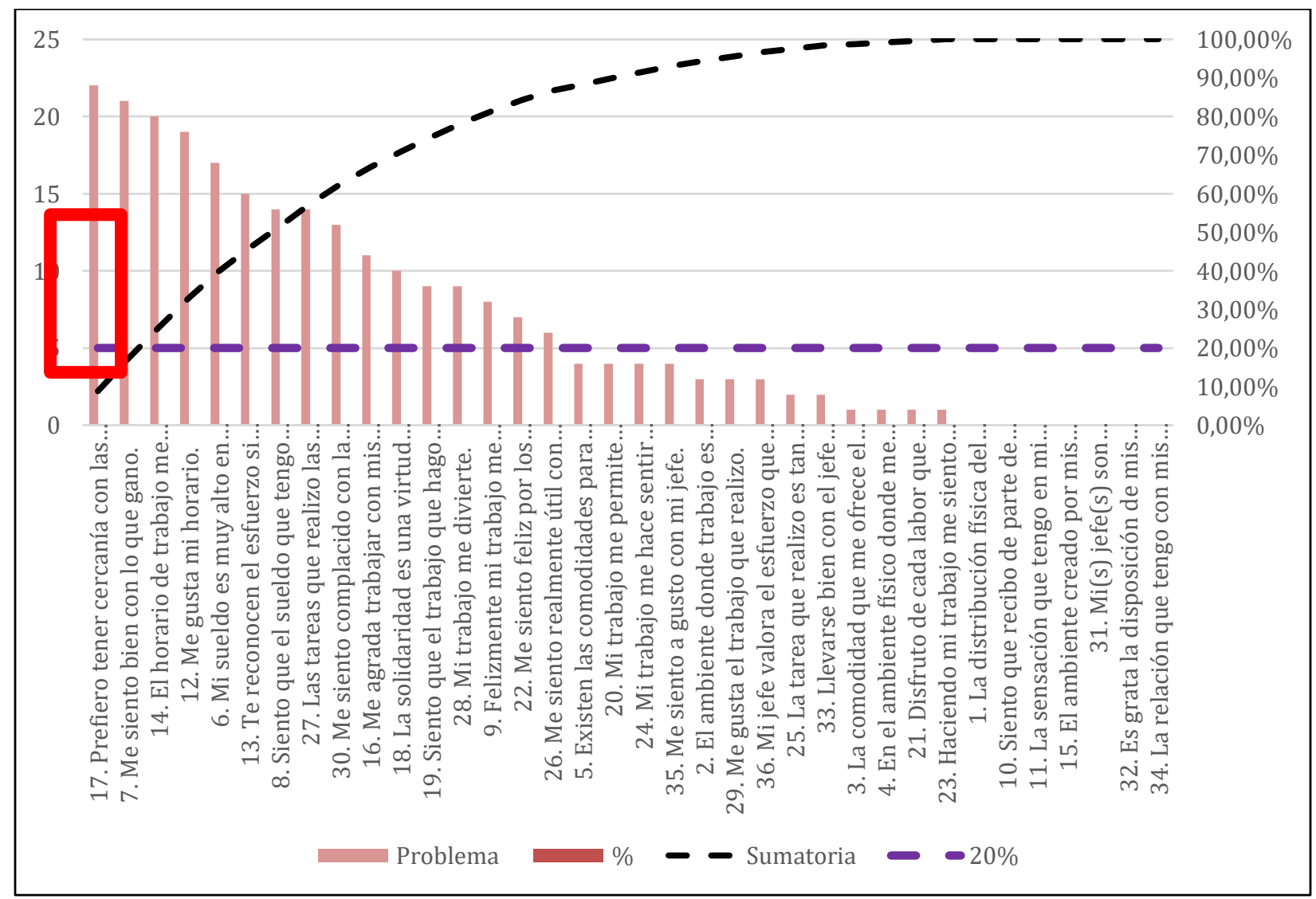


En la figura 1 correspondiente al diagrama de Pareto se visualizan los resultados de las preguntas realizadas a los encuestados, donde se pudo identificar 2 preguntas críticas que originan el 80\% de los problemas; la primera causa la determinó la pregunta $\mathrm{N}^{\circ} 17$, ¿Prefiero tener cercanía con las personas con las que trabajo?, la cual pertenece a la subcategoría relaciones sociales, dicha pregunta obtuvo como resultado que la suma de las respuestas de nunca y casi nunca daban más del 70.00\%; se evidencia la carencia de confraternización, debido que al no desear entablar una relación cercana entre compañeros, se provocan estragos en el equipo de trabajo al no haber cohesión y ser desunidos, perjudicando a la empresa. La segunda causa la estableció la pregunta $\mathrm{N}^{\circ}$ 7, ¿Me siento bien con lo que gano?, dicha pregunta correspondiente a la subcategoría beneficios laborales y/o remunerativos, obtuvo un mayoritario casi nunca, lo que constituye un nivel de insatisfacción por parte de los trabajadores respecto al salario percibido.

\section{Figura 2}

\section{Análisis cualitativo de la categoría satisfacción laboral}



Fuente: Elaboración propia 
Del análisis cualitativo realizado en el área administrativa de una empresa importadora se pudo identificar tres principales problemas con relación a la satisfacción laboral; el primer problema es la remuneración no acorde con las tareas realizadas, debido a que muchos de los colaboradores no han sido beneficiados con un aumento de sueldo en varios años, a esto se suma la molestia de saber que las labores que ejecutan son remuneradas de mejor forma en las compañías competidoras; sin embargo, pese a no sentirse conformes, tienen temor de migrar de trabajo, pero esta insatisfacción repercute sobre el rendimiento que la empresa calcula recibir por parte de sus empleados.

El deterioro de las relaciones humanas es el segundo problema identificado; a raíz de los cambios que produjo la pandemia sobre el confinamiento y la necesidad del trabajo remoto, los trabajadores se vieron afectados al disminuir la interacción con sus compañeros más cercanos; si bien es cierto, la relación entre el personal no era muy unida, si podía percibirse lazos de amistad entre cada sub-grupo o sub-área de trabajo, más no en su conjunto. Por último, el tercer problema es la carencia de reconocimiento al mérito individual; los trabajadores manifestaron su deseo de ser felicitados por su buen desempeño, ser valorados y reconocer su esfuerzo, muy a pesar que la empresa pueda brindar algún bono por una condición especial, lo concreto es que no existe ninguna distinción individual que premie al que mejores resultados logra obtener. Asimismo, al trabajar de forma remota, no se contabilizan las horas extras que se emplean en completar una tarea, debido a que por el tipo de trabajo algunos se ven en la necesidad de utilizar más horas para la finalización de una labor, pero sin recibir compensación por ello.

\section{Propuesta de mejora de la satisfacción laboral}

Para esta investigación, se plantearon tres objetivos; en primer lugar, se consideró formar equipos de trabajo de élite que contribuyan a la empresa, para ello, se contempla realizar un estudio del mercado salarial actual entre las compañías del sector, con dicha información se podrá elaborar la escala remunerativa y el informe que servirá para su implementación; seguidamente, se evaluará a todos los trabajadores para medir sus habilidades utilizando los lineamientos indicados en el manual de cómo identificar las habilidades de un colaborador; una vez obtenidos los resultados se podrá reubicar al personal en función a sus capacidades, con el propósito de desarrollar en ellos la pasión laboral. Por otra parte, los indicadores que servirán para controlar el avance y eficacia de las actividades empleadas en este objetivo son: 
Figura 3

Esquema de indicadores del objetivo 1

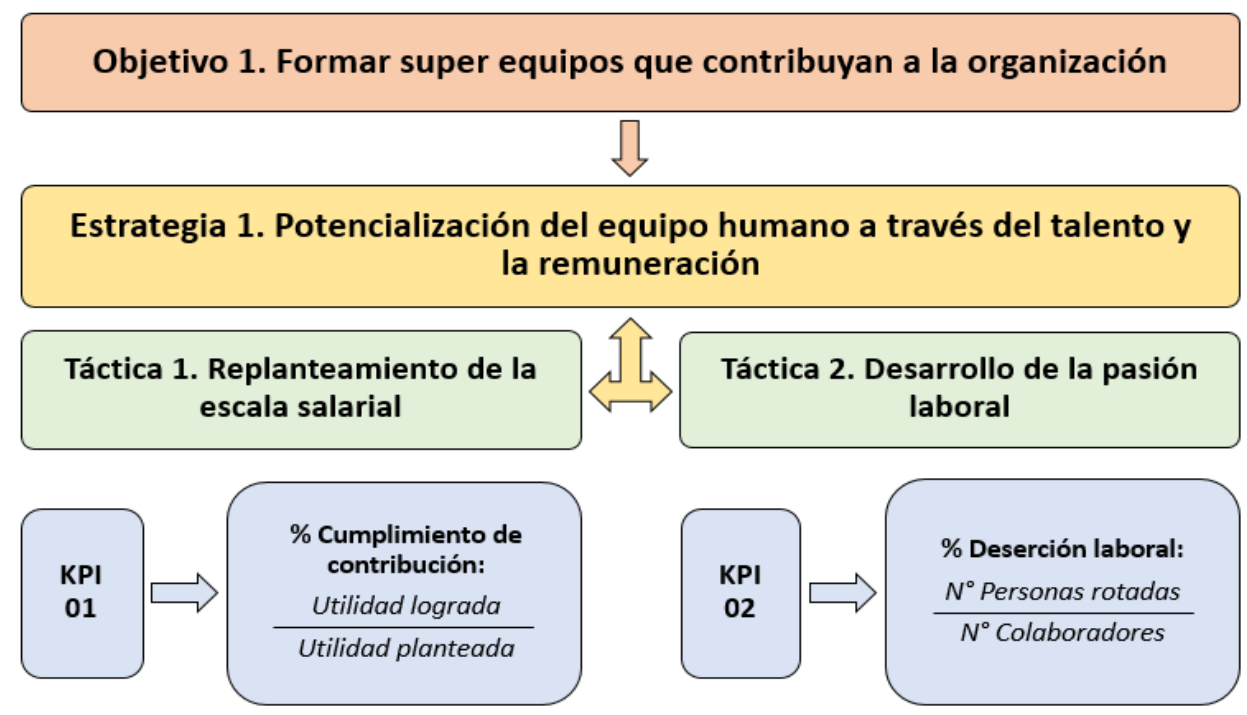

Fuente: Elaboración propia.

Este primer objetivo, tiene actividades programadas con una duración de 120 días y un presupuesto asignado de $\mathrm{S} / 1000.00$ como se indica en la tabla 3:

\section{Tabla 3}

Egresos objetivo 1

\begin{tabular}{llrcr}
\hline N & Descripción & P.Unit & Cant. & P.Total \\
\hline 1 & Asesoría de agencia especializada & 300.00 & 1 & 300.00 \\
2 & Revisión legal del diseño & 50.00 & 1 & 50.00 \\
3 & Asesoría psicológica para la evaluación de habilidades & 250.00 & 1 & 250.00 \\
4 & Gastos logísticos y entrega de materiales adicionales & 100.00 & 1 & 100.00 \\
5 & Capacitaciones virtuales por sub áreas & 50.00 & 6 & 300.00 \\
\hline
\end{tabular}

Total de egresos

S/ $1,000.00$

Fuente: Elaboración propia.

En el segundo objetivo, se busca fortalecer las relaciones humanas a través de un manual que permita afianzar la comunicación asertiva, la empatía, el liderazgo y la capacidad de interrelacionarse entre compañeros, para ello, este manual contempla ocho talleres con su respectiva guía de instrucciones, para poner en marcha las actividades de aprendizaje 
organizacional en equipos de trabajo, juego de roles, comunicación asertiva, liderazgo, conflictos, manejo de cambios, resolución de conflictos, debate y participación efectiva. Adicionalmente se incluirá en el reglamento de trabajo, el fomento a la cultura de la sana amistad en la empresa; asimismo, se medirá el avance de los talleres a través de encuestas de satisfacción del cliente interno. Por otra parte, los indicadores que servirán para controlar el progreso y eficacia de las actividades empleadas en este objetivo son:

\section{Figura 4}

Esquema de indicadores del objetivo 2

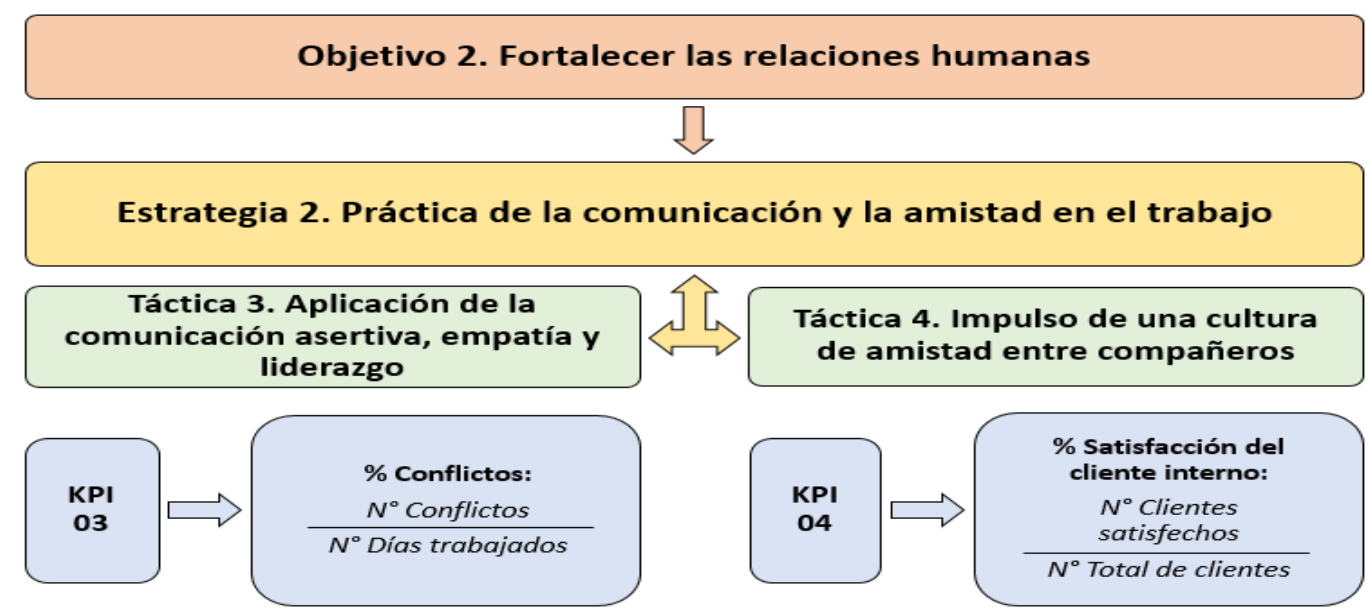

Fuente: Elaboración propia.

En este caso, las actividades programadas tendrán una duración de 86 días, y contarán con un presupuesto asignado de $\mathrm{S} / 500.00$ según detalle mostrado en la tabla 4:

\section{Tabla 4}

Egresos objetivo 2

\begin{tabular}{llrcc}
\hline N & Descripción & P.Unit & Cant. & P.Total \\
\hline 1 & Asesoría técnica & 150.00 & 1 & 150.00 \\
2 & Materiales para el taller & 1.00 & 150 & 150.00 \\
3 & Asesoría de proyecto & 200.00 & 1 & 200.00 \\
\hline Total de egresos & & & S/ 500.00 \\
\hline
\end{tabular}

Fuente: Elaboración propia.

Por último, el tercer objetivo es el de instaurar el reconocimiento al mérito individual, para ello, se desarrollará un modelo de aplicación y un manual detallado con las bases y aspectos a 
Propuesta de mejora de la satisfacción laboral en el área administrativa de una empresa importadora en Lima (2020)

tomar en cuenta para brindar un salario emocional que sea justo y que tenga como principio la productividad del colaborador; de igual forma, el avance será medido a través de la encuesta de satisfacción laboral que se debe realizar periódicamente. Por otro lado, en cuanto a los indicadores que facilitarán la medición y control de las actividades desarrolladas en este objetivo son:

\section{Figura 5}

Esquema de indicadores del objetivo 3

Objetivo 3. Implementar el reconocimiento al mérito individual

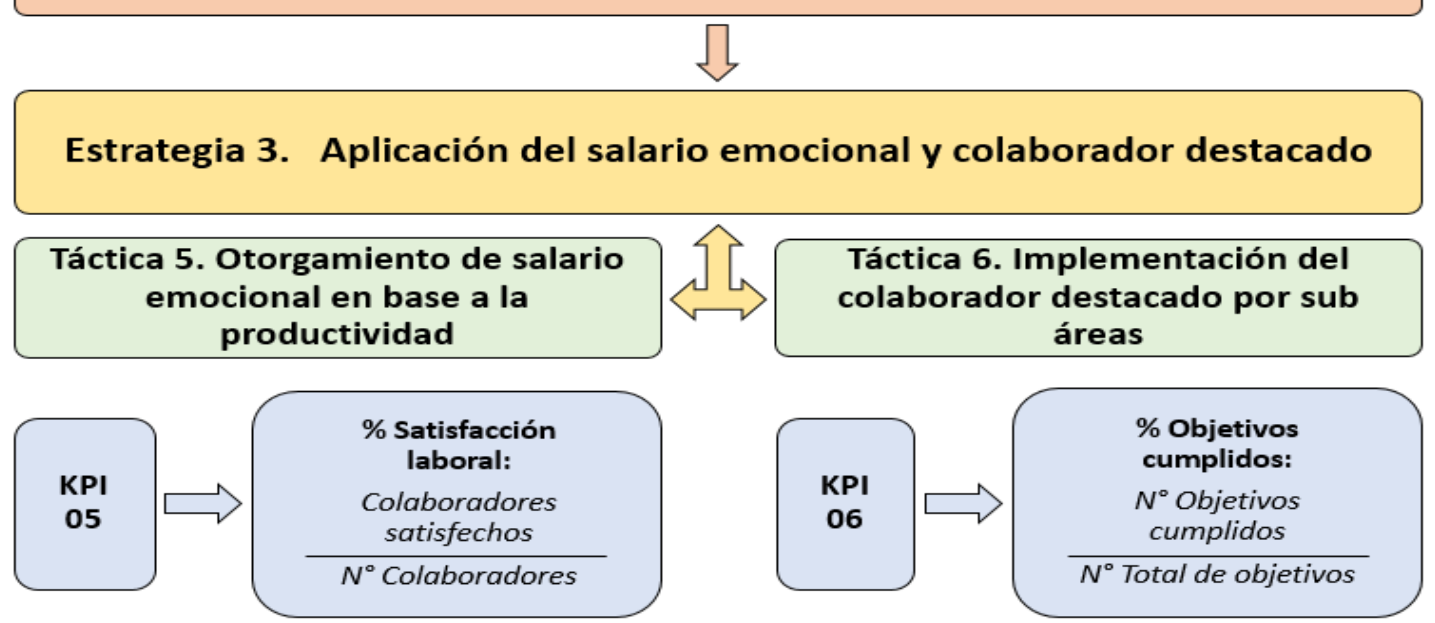

Fuente: Elaboración propia.

Las actividades tendrán una duración de 81 días y un presupuesto asignado de S/ 2880.00 con el detalle indicado según la tabla 5:

\section{Tabla 5}

Egresos objetivo 3

\begin{tabular}{lcccc}
\hline $\mathbf{N}$ & Descripción & P.Unit & Cant. & P.Total \\
\hline 1 & Vales para personal destacado durante 12 meses & 20.00 & 144 & $2,880.00$ \\
& & & S/ $2,880.00$ \\
\hline Total de egresos & & & \\
\hline
\end{tabular}

Fuente: Elaboración propia.

\section{Discusión}

El presente estudio se desarrolló con la finalidad de indagar cómo se hallaba la satisfacción laboral dentro del área administrativa en una empresa importadora, de esta forma, poder determinar 
cuáles eran los problemas que causaban impacto en los trabajadores y así determinar una propuesta de mejora consistente en varias herramientas, talleres y otras actividades que corrigiesen y mejoraran la actual situación en dicha empresa. En igual modo, posterior al diagnóstico de la categoría satisfacción laboral y de las siete subcategorías que la conforman, se encontraron tres problemas de mayor relevancia y ellos son: la remuneración no coherente con las labores realizadas, el fraccionamiento de las relaciones humanas y la falta de reconocimiento al mérito individual.

En cuanto a la subcategoría condiciones físicas y/o materiales, la misma que es entendida como aquellos componentes ambientales, tecnológicos y de asignación de espacios que generen un impacto favorable o no sobre el bienestar de los trabajadores (López y Moscoso, 2018; Gómez, 2015). Asimismo, se pudo hallar que el $66.7 \%$ indica que casi siempre su lugar de trabajo es insuperable; de similar forma, el $56.7 \%$ encontró que su ambiente de trabajo era casi siempre placentero. Por otro lado, debido a la pandemia, la empresa le ha dotado al personal enviándole a sus domicilios, equipos, mobiliario y herramientas de soporte; en este sentido, Bollet y Flores, (2018) establecieron que, existiendo una buena calidad en el ambiente laboral, los trabajadores desarrollarán un grado muy alto en cuanto a satisfacción laboral se refiere, incidiendo directamente en la mejora de la productividad en la empresa. Todo ello, permite indicar que esta subcategoría fue evaluada satisfactoriamente.

En relación a la subcategoría beneficios laborales y/o remunerativos, es de importancia precisar que no hace referencia exclusiva al dinero que se recibe a cambio del cumplimiento de una tarea asignada, sino también incluye en la actualidad los beneficios otorgados como por ejemplo los bonos, los cuales son percibidos como un valor agregado y generan una repercusión positiva en la motivación del colaborador (Reaño, 2019). Por otra parte, en cuanto a los resultados se determinó que el $60.0 \%$ casi nunca se siente bien con lo que percibe por sueldo; asimismo, se puso al descubierto que un gran número de empleados no ha recibido un incremento salarial en varios años, poniendo claramente en evidencia el descontento actual en esta materia. De igual forma, en relación a la situación vista, Dos Santos, Dias, Calvancanti, y Coutinho (2016) afirmaron en su estudio que el contar con una remuneración acorde a la labor desempeñada, es un factor que impacta en el grado de satisfacción de los trabajadores brasileños. En concordancia con ello, Rosales, Rodal, Chumbi, y Buñay (2017) aseguraron que existe un nivel de correlación muy alto entre la satisfacción laboral y el salario percibido. Por todo lo indicado, se pudo concluir que en esta subcategoría se presenta una seria deficiencia que repercute en la satisfacción de los empleados.

Con respecto a la subcategoría políticas administrativas, se pueden entender como las normas para dirigir, controlar y regular la forma en que actúa una organización con sus respectivos grupos de interés; por otro lado, se encuentran conectadas con la misión institucional, de ahí deriva la importancia de su comprensión y aplicación (Flores, 2015; Cardoza, Bermúdez y Zapata, 2019; Ávila, 2019). En cuanto a los resultados, se encontró que el $40.0 \%$ no está conforme con su horario de trabajo, de igual forma, un $36.7 \%$ señala que las horas extras no son reconocidas, y esto ocurre desde el cambio de modalidad de presencial a remoto a raíz de la pandemia. En ese sentido, Bendezú-Pacífico (2020) logró demostrar la existencia de un nivel de correlación entre la satisfacción laboral y la gestión del talento humano, debido a que, por medio del comportamiento organizacional, se consideran las normas de la empresa, la cultura, la comunicación, la motivación, 
Propuesta de mejora de la satisfacción laboral en el área administrativa de una empresa importadora en Lima (2020)

la compensación laboral y el trabajo coordinado en equipo. Todo lo anteriormente indicado, permitió ratificar que la empresa no cuenta con una normativa que solucione el impase de las horas extras no consideradas.

En cuanto a la subcategoría relaciones sociales, se puede señalar que es importante mantenerlas en un nivel apropiado debido a la necesidad que tienen los trabajadores por generar lazos de confraternidad con sus compañeros, superiores y en general con toda aquella persona que trabaja en la empresa, de esta manera el individuo se sentirá aceptado por los demás (Reaño, 2019; Ávila, 2019; Ramos, 2015). Por otra parte, es preciso indicar que en la organización materia de estudio, el $36.7 \%$ nunca preferiría tener una relación cercana con sus compañeros, poniendo en evidencia una ruptura en las relaciones sociales, con mayor razón, considerando que el 33.3\% mencionó que casi nunca le es de agrado trabajar con sus colegas. Además, esta situación empeoró desde que se trabaja desde casa, al no tener ningún tipo de interacción entre ellos, originando la falta de apoyo entre compañeros y el deterioro en la calidad de atención a nivel interno. De igual forma, en concordancia con lo manifestado por Dos Santos, Dias, Calvancanti, y Coutinho (2016) los que señalaron que un factor preponderante en la motivación y por ende a la satisfacción laboral, era llevar una buena relación con sus compañeros de trabajo.

En referencia a la subcategoría desarrollo personal, es posible aseverar que ésta comprende las experiencias absorbidas y difundidas por la organización, otorgando así, la ventaja a cada individuo para que pueda mejorar su rendimiento u obtener el desarrollo profesional anhelado (Ávila, 2019; Jiménez, 2015; Navarro, 2018). Por otra parte, los resultados del estudio arrojaron que solo el $30.0 \%$ de encuestados disfruta su trabajo, asimismo, se encontró que la empresa les brinda capacitaciones, pero solo de seguridad y salud en el trabajo, presentándose una carencia de adiestramientos específicos para cada función. En relación al tema, sostuvieron que se realizan convocatorias internas para acceder a vacantes disponibles, brindándole opciones de crecimiento profesional a cada uno de sus colaboradores. Sobre el particular, en otro estudio efectuado por Pujol-Cols y Foutel (2019), identificaron que la satisfacción laboral influye en la salud mental y a su vez, la salud mental repercute en la salud física, para ello, comprobaron que en ambientes donde las decisiones importantes eran tomadas con plena libertad y autonomía, los trabajadores conseguían una mejora en su condición mental y física, aumentando incluso su desempeño laboral.

Sobre la subcategoría desempeño de tareas, se entiende como la resultante de combinar destrezas, talentos, experticia y habilidades que cada trabajador posee, así como su propia personalidad, todo ello, marcará la pauta sobre las funciones que realicen y que tan productivos puedan llegar a ser (Trelles y Varas, 2017; Guzmán, 2017; Ávila, 2019). De otra parte, en las encuestas realizadas se obtuvo que el 53.3\% de colaboradores señalan que sus labores siempre son de importancia, lo que se ratifica con el $43.3 \%$ que indica sentirse muy útil con las tareas que les son asignadas; sin embargo, en contraposición con este último resultado, el 33.3\% señaló que casi nunca es de importancia la labor que realizan, debido a que se sienten con la capacidad de resolver encargos de mayor exigencia intelectual y de destreza. Lo descrito toma mayor sentido cuando se analiza lo explicado por Bargsted, Ramírez-Vielma, y Yeves, (2019) quienes concluyeron en su investigación que, para conseguir la satisfacción laboral, los trabajadores deben contar con autonomía para desarrollar sus funciones, que las labores encomendadas sean de importancia y que valoren su desempeño. Por esta razón, es que se considera a esta subcategoría como punto esencial para mejorar la satisfacción y productividad de los empleados en favor de la empresa. 
En lo concerniente a la subcategoría relación con la autoridad, la cual, hace alusión a las condiciones laborales del colaborador en relación con el deseo de efectuar las indicaciones impartidas por sus supervisores o jefaturas; de igual modo, los trabajadores puedan contar con la facilidad de tener una estrecha relación jefe-subordinado (Oliveros, Fandiño y Torres, 2016; Rodríguez, Núñez y Cáceres, 2010; Ávila, 2019). En cuanto a los resultados, se obtuvo que el $83.3 \%$ mantiene una cordial relación con todos sus superiores, en especial, porque indican que los líderes de la organización siempre los escuchan y les dan plena libertad para que utilicen su capacidad y criterio en la resolución de problemas. Por otra parte, vale mencionar lo estudiado por Nolazco, Ávila, Chávez, Sicheri, y Carhuancho, (2020), quienes señalan que el desinterés de las jefaturas hacia su personal, está directamente asociado a la carencia de diálogo con ellos, causando un alto impacto en la satisfacción laboral. Estos resultados permiten concluir que esta empresa importadora mantiene una muy buena relación entre los colaboradores y sus jefaturas, situación que con el tiempo ha ido mejorando.

\section{Conclusiones}

Luego de concluir con la investigación realizada, se identificó que los factores que afectan la satisfacción laboral son la remuneración no acorde con las labores realizadas, el deterioro en las relaciones humanas y la carencia de reconocimiento al mérito. De igual forma, se pudo diagnosticar que la subcategoría beneficios laborales y/o remunerativos y la subcategoría relaciones sociales, son las que cuentan con mayor número de observaciones negativas, afectando la satisfacción laboral. En consecuencia, con lo analizado, se propone este modelo integral con el propósito de mejorar la satisfacción laboral en el área administrativa de una empresa importadora; asimismo, este modelo planteado contiene actividades, talleres de aprendizaje, diversos manuales y métodos de reconocimiento, los cuales en conjunto son necesarios para elevar el rendimiento del personal, a través de multiplicar su gusto y satisfacción por el trabajo.

Por otra parte, es recomendable priorizar las acciones que brinden solución a las causas que afectan la satisfacción laboral; en especial de ser pertinente, aplicar más talleres prácticos para mejorar las relaciones humanas en la organización. De igual manera, se recomienda la activación de un control constante de parte de la empresa, donde se actúe con prontitud sobre las subcategorías que reporten deficiencias, de esta forma se puede tomar las medidas correctivas en el modelo planteado y reformular las actividades correspondientes. Por último, se recomienda la ejecución inmediata de este modelo integral para transformar la situación de la empresa y que se vea beneficiada al contar con un excelente equipo de trabajo, muy motivado y satisfecho con su labor, en condiciones justas y prestas a dar lo mejor de sí mismos para cumplir los objetivos de la organización.

\section{Referencias Bibliográficas}

Ávila, J. (2019). Las 3S para mejorar la satisfacción laboral del área de producción en una empresa metalmecánica, Lima 2019. [Tesis de licenciatura. Universidad Privada Norbert Wiener, Lima. http://repositorio.uwiener.edu.pe/handle/123456789/3546 
Propuesta de mejora de la satisfacción laboral en el área administrativa de una empresa importadora en Lima (2020)

Bargsted, M., Ramírez-Vielma, R., y Yeves, J. (2019). Autoeficacia profesional y satisfacción laboral: el papel mediador del diseño del trabajo. Revista de Psicología del Trabajo y de las Organizaciones, 157-163. https://doi.org/10.5093/jwop2019a18

Bendezú-Pacífico, K. (2020). Gestión del talento humano y la satisfacción laboral de los trabajadores de una institución educativa privada. Investigación Valdizana, 22-28. https://doi.org/10.33554/riv.14.1.494

Bernal, C. (2010). Metodología de la investigación, administración, economía, humanidades y ciencias sociales. (Tercera ed.). Bogotá: Pearson Educación de Colombia Ltda.

Bollet, F., y Flores, E. (2018). Satisfacción laboral y desempeño de las personas en la empresa Protege Oriente S.A., Pucallpa, 2018. Revista de investigación científica cultura viva amazónica, 42-47. https://doi.org/10.37292/riccva.v3i03.121

Cardoza, A., Bermúdez, F., y Zapata, R. (s.f.). Administración: Cultura organizacional. (Tesis de licenciatura). Universidad Nacional Autónoma de Nicaragua, Managua.

Carhuancho, I., Nolazco, F., Sicheri, L., Guerrero, M., y Casana, K. (2019). Metodología para la investigación holística. Ecuador: UIDE. https://repositorio.uide.edu.ec/handle/37000/3893

Chiavenato, I. (2006). Introducción general a la teoría de la administración (Séptima ed.). México: McGraw HIll / Interamericana Editores, S.A. de C.V.

Chiavenato, I. (2007). Administración de recursos humanos. El capital humano de las organizaciones (Octava Edición ed.). México: McGraw Hill / Interamericana Editores, S.A. de C.V.

Chiavenato, I. (2009). Comportamiento organizacional. La dinámica del éxito en las organizaciones. (Segunda Edición ed.). México: McGraw Hill / Interamericana Editores, S.A. de C.V.

Dávila, C. (2001). Teorías organizacionales y administración. Enfoque crítico. (Segunda ed.). Colombia: McGraw Hill Interamericana, S.A.

Del Cid, A., Méndez, R., y Sandoval, F. (2011). Investigación. Fundamentos y metodología. (Segunda ed.). México: Pearson Educación de México, S.A. de C.V.

Dos Santos, E., Dias, S., Calvancanti, M., \& Coutinho, T. (2016). Percepção de responsabilidade social e satisfação no trabalho: um estudo em empresas brasileiras. REGE - Revista de Gestão, 306-315. https://doi.org/10.1016/j.rege.2016.09.006

Flores, S. (2015). Proceso administrativo y gestión empresarial en Coproabas, Jinotega. (Tesis de maestría). Universidad Nacional Autónoma de Nicaragua, Matagalpa.

Gómez, R. (2015). Condiciones de trabajo y salud en el sector de la construcción, ¿cuestión de jerarquías? [Tesis Doctoral. Universidad Pública de Navarra, Pamplona]. https://academica-e.unavarra.es/handle/2454/20948

Guzmán, B. (2017). Desempeño laboral y dimensiones de personalidad en los trabajadores de la Municipalidad Provincial del Santa, Chimbote-2017. [Tesis de licenciatura. Universidad San Pedro, Chimbote].

Hernández, R., Fernández, C., y Baptista, M. (2014). Metodología de la investigación. México: McGraw-Hill / Interamericana Editores, S.A. de C.V.

Hurtado, J. (2000). Metodología de la investigación holística (Tercera ed.). Caracas: Fundación Sypal servicios y proyecciones para America Latina.

Jiménez, E. (2015). Desarrollo personal y profesional de maestras de educación primaria: Aportes de la orientación. Revista Actualidades Investigativas en Educación., 1-29. https://doi.org/10.15517/aie.v15i1.17624

Esta obra se comparte bajo la licencia Creative Common Atribución-No Comercial 4.0 International (CC BY-NC 4.0) Revista de la Universidad Internacional del Ecuador. URL: https://www.uide.edu.ec/ 
Lee Hecht Harrison - DBM Perú. (2018). Empleabilidad y mercado laboral. Perú: LHH-DBM Perú.

Locke, E.A. (1976). The Nature and causes of job satisfaction, in Dunnette. Handbook of industrial and organizational psicology. Chicago. United States: Rand McNally College.

López, C., y Moscoso, G. (2018). Condiciones de trabajo relacionadas al nivel de fatiga en el personal de enfermería del servicio de emergencia del hospital Leopoldo Barton - Essalud, 2018. [Tesis de licenciatura. Universidad Privada Norbert Wiener, Lima].

Maslow, A. (1991). Motivación y personalidad. España: Ediciones Díaz de Santos, S.A.

Navarro, T. (2018). Desarrollo personal y desempeño laboral en la Caja Municipal de ahorro y crédito Cusco S.A., agencia Andahuaylas-2016. [Tesis de licenciatura. Universidad Nacional José María Arguedas, Andahuaylas].

Nolazco, F., Ávila, J., Chávez, W., Sicheri, L., \& Carhuancho, I. (2020). Job satisfaction in the area of production in a metalworking company. International Journal of Scientific \& Technology Research, 2711-2715.

Oliveros, J., Fandiño, J., y Torres, Y. (2016). Factores motivacionales hacia el trabajo en el departamento de enfermería de un hospital de cuarto nivel en la ciudad de Bogotá. Repertorio de Medicina y Cirugía., 118-125. https://doi.org/10.1016/j.reper.2016.03.004

Pujol-Cols, L., y Foutel, M. (2019). Satisfacción laboral y salud: Un análisis de efectos directos e indirectos en gerentes argentinos. Cuadernos de investigación, 32-59. https://doi.org/10.11144/Javeriana.cao32-59.slsaed

Ramos, V. (2015). Relaciones Sociales en el trabajo y satisfacción laboral. (Tesis de licenciatura). Universidad Casa Grande, Guayaquil.

Randstad Workmonitor. (2019). La satisfacción laboral en España cae tres puntos en un año y se sitúa por debajo de la media europea. España: Randstad Workmonitor.

Reaño, R. (2019). Relación entre las condiciones laborales y la satisfacción laboral del personal administrativo de la administración central de la Universidad Nacional de San Agustín de Arequipa 2018. (Tesis de maestría). Universidad Nacional de San Agustín de Arequipa, Arequipa.

Rodríguez, D. (2019). Estrategias para retener el talento humano en una empresa de servicios generales, Lima 2019. (Tesis de licenciatura). Universidad Privada Norbert Wiener, Lima.

Rodríguez, D., Núñez, L., y Cáceres, A. (2010). Estudio comparativo de la satisfacción laboral universitaria en el núcleo de Anzoátegui de la Universidad del Oriente. Revista Investigación y Postgrado, 63-80.

Ronald Carrer Services Group, \& Andina agencia peruana de noticias. (2018). Día del trabajo: el 74\% de peruanos cambaría de empleo para ser más feliz. Perú: Andina agencia peruana de noticias.

Rosales, G., Rodal, A., Chumbi, V., y Buñay, R. (2017). Análisis de la satisfacción laboral y desempeño académico profesional del estudiantado graduado en Psicología de la Universidad de Cuenca, Ecuador. Revista Electrónica Educare, 1-24. http://doi.org/10.15359/ree.21-3.13

Sap Consultoria. (2016). Pesquisa home office Brasil 2016. Brasil: Sap Consultoria.

Sanchez, M., y García, M. (2017). Satisfacción laboral en los entornos de trabajo. Una exploración cualitativa para su estudio. Scientia Et Technica, 161-166.

Society for human resource management. (2017). Employee job satisfaction and engagement: The doors of opportunity are open. Estados Unidos: Society for human resource management. 
Propuesta de mejora de la satisfacción laboral en el área administrativa de una empresa importadora en Lima (2020)

Tecla, A., y Garza, A. (1981). Teoría, métodos y técnicas en la investigación social. San Salvador: Ediciones del taller abierto.

Trelles, J., y Varas, A. (2017). Relación entre el clima laboral y el desempeño de los colaboradores de la agencia distribuidora servicios y representaciones dial S.R.L., en la ciudad de Trujillo, en el año 2017. [Tesis de licenciatura. Universidad Privada Antonio Orrego, Trujillo]. 